\title{
LAGUNA-LBNO: a very long baseline neutrino oscillation experiment
}

\author{
Silvestro Di Luise ${ }^{* \dagger}$ \\ ETH Zurich Institute for Particle Physics \\ E-mail: silvestro.di.luisedcern.ch
}

LAGUNA (Large Apparatus for Grand Unification and Neutrino Astrophysics)-LBNO(LongBaseline Neutrino Oscillation)[1, 2] is the proposal for a long baseline neutrino oscillation experiment with a new conventional neutrino beam aimed at a next generation deep-underground neutrino observatory composed of a double phase liquid Argon detector and a magnetized iron calorimeter located at the Pyhäsalami mine, $2300 \mathrm{~km}$ from CERN. The design of experimental apparatus is mainly driven by the successful phase of of $R \& D$ and prototyping on small scale setups. An unprecedented rich physics program can be pursued.

- neutrino oscillations can be studied in detail fully exploiting, for the first time, the energy spectrum information of the oscillation probability (L/E method) in appearance and disappearance mode at long baseline. An exposure of $2.25 \times 10^{20}$ protons on target (p.o.t.) from the CERN SPS at $400 \mathrm{GeV}$ would allow for a definitive determination (> $5 \sigma$ C.L.) of the neutrino mass hierarchy for any value of the $\mathrm{CP}$ violation phase $\delta_{C P}$. The $\mathrm{CP}$ violation can be instead discovered at $3 \sigma$, through the L/E method, with a coverage of the $70 \%$ of the $\delta \mathrm{CP}$ parameter space, providing $1.5 \times 10^{21}$ p.o.t., which is achievable possibly in 10 years of exposure. Grand Unified Theories (GUT) can be investigated with searches for several nucleon decay signatures and with unprecedented sensitivities thanks to the very large detector mass and the extremely clean cosmological background.

- atmospheric neutrinos, as well as unknown astrophysical neutrinos (e.g. from D.M. annihilation) can be studied for the first time over a wide range of energies and final states

- the explosion of a galactic Supernova (SN) will be detected with large statistics, in neutrino and antineutrino modes and in all flavors allowing to constrain both the astrophysics of the SN and the neutrino flavor oscillations.

It will be highlighted the physics reach of a CERN-Pyhäsalmi long baseline conventional beam, assuming the existing CERN SPS performance and a far detector with a fiducial mass of $20 \mathrm{kton}$.

36th International Conference on High Energy Physics

4-11 July 2012

Melbourne, Australia

* Speaker.

${ }^{\dagger}$ A footnote may follow. 
In the year 2011 several experiments have finally measured the last unknown neutrino mixing angle $\theta_{13}$ to be different form zero[3]. Thanks to these experiments, the value of $\theta_{13}$ is now known with a precision of about $15 \%$, equivalent to the knowledge of the other mixing angles. The discovery that all three mixing angles $\theta_{12}, \theta_{23}$ and $\theta_{13}$ parametrizing the Pontecorvo-MakiNakagawa-Sakata (PMNS) leptonic matrix are non-vanishing and large, has ascertained the $3 \times 3$ unitary character of the PMNS matrix and opened the possibility of its non-trivial complex nature. This raises the intriguing possibility that neutrinos (or their heavy neutrino partners) might have played an important role in the early age of the Universe contributing to the creation of the baryon asymmetry which is responsible today for the matter dominance. To observe $\mathrm{CP}$ asymmetry in the leptonic sector is therefore among the most important topics in Particle Physics today. To resolve the question of CP requires a definite determination of the neutrino mass hierarchy, since matter effects also introduce a different oscillatory behavior between neutrino and antineutrinos when crossing through the Earth, determined by an effective potential which changes sign between the normal and inverted hierarchy. These arguments strongly advocate a further exploration of neutrinos, and indeed in a more urgent and prominent way, but yet also more accessible given the large value of $\theta_{13}$.

In the oscillation probability the CP-violating effects of $\delta_{C P}$ are modulated by those of all three mixing angles and their interplay, resulting in complicated dependencies and leading to an a priori eight-fold parameter degeneracy[4]. In addition, the situation in all long baseline experiment is complicated by the matter effects. Overall, it is known that the energy dependence of the probability can resolve several of these issues and allows in particular disentangling the CP-driven and the matter-driven effects, if the baseline is large enough. In this context, it is instructive to define two asymmetries between the probability of oscillations of neutrinos and antineutrinos, one related to $\mathrm{CP}$ effect in vaccum

$$
A_{C P}^{v a c}\left(\delta_{C P}\right)=\frac{\left|P^{v a c}(v)-P^{v a c}(\bar{v})\right|}{\left|P^{v a c}(v)+P^{v a c}(\bar{v})\right|}
$$

and the other to the matter effects computed in matter for a fixed value of $\delta_{C P}$ ( $\rho$ is the traversed Earth matter density, assumed constant)

$$
A_{C P}(\rho)=\frac{\left|P^{\text {mat }}(v)-P^{\text {mat }}(\bar{v})\right|}{\left|P^{\text {mat }}(v)+P^{\text {mat }}(\bar{v})\right|}
$$

These two variables, plotted in the two dimensional plane of the neutrino energy $E_{v}$ versus the baseline $L$, are shown in Fig. 1. In these graphs, the darker regions correspond to combinations of neutrino energy and baseline at which the oscillation phenomena is not sensitive to the effect, while the light regions corresponds to those where the effect is larger. It can be noted that:

- The CP asymmetries are largest at the $2^{\text {nd }}, 3^{\text {rd }}$, etc. maxima.

- The matter asymmetry covers a broad region just below and dominates around the $1^{\text {st }}$ maximum.

- Longer baselines and wide-band beams to cover several maxima are needed to resolve degeneracies. 

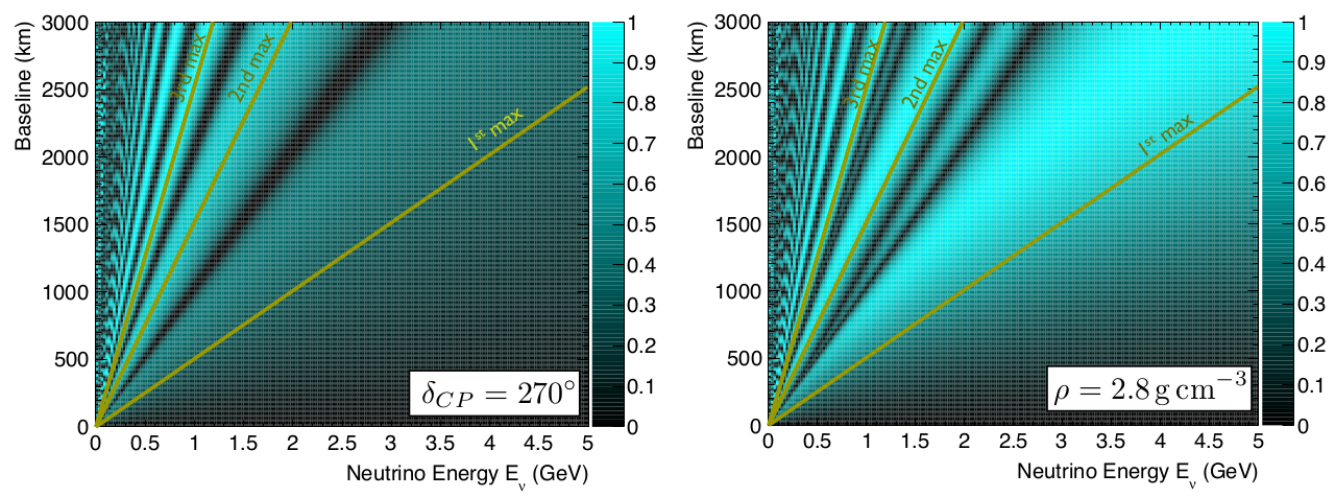

Figure 1: The two asymmetries $A_{C P}^{v a c}\left(\delta_{C P}\right)$ and $A_{C P}(\rho)$ in the $\left(E_{v}, L\right)$ plane. Superimposed lines indicate the $1^{\text {st }}, 2^{\text {nd }}$ and $3^{\text {rd }}$ oscillation maxima at constant $L / E_{v}$.

- Assuming a reasonable energy threshold of $500 \mathrm{MeV}$, which is a realistic value taking into account realizable conventional neutrino beam fluxes, and the vanishing neutrino cross-sections at low energies (in particular for antineutrinos), the measurement of the $2^{\text {nd }}$ maximum requires a baseline greater than $1000 \mathrm{~km}: E_{v}^{2 n d-m a x}>0.5 \mathrm{GeV}$ which means $L>1000 \mathrm{~km}$.

If the distance between source and detector is fixed, the oscillatory behavior can be easily translated to that for the expected neutrino energy spectrum of the oscillated events. If the neutrino energy spectrum of the oscillated events can be reconstructed with sufficiently good resolution in order to distinguish first and second maxima, the spectral information obtained is invaluable for the unambiguous determination of the oscillation parameters. LBNO adopts a combination of methods, by precisely measuring the disappearance and appearance energy spectrum shapes (in particular, peak position and height for $1^{\text {st }}$ and $2^{\text {nd }}$ oscillation maximum and minimum) with high resolution, and by comparing neutrino- and antineutrino-induced oscillations. The liquid Argon and magnetized iron detectors will in fact provide complementary studies of all three active transitions $v_{\mu} \rightarrow v_{\mu}$, $v_{\mu} \rightarrow v_{e}$ and $v_{\mu} \rightarrow v_{\tau}$ over the optimized range of neutrino energies. A precise investigation of the oscillation probabilities as a function of energy and a comparison of neutrino and antineutrino behaviors will verify if they follow the expectations from 3-generation neutrino mixing. These measurements will also yield a definitive resolution of the neutrino mass hierarchy (MH) and a significant exploration of CP-violation (CPV) in the neutrino sector. The $2300 \mathrm{~km}$ baseline is adequate to have an excellent separation of the asymmetry due to the matter effects (i.e. the mass hierarchy measurement) and the $\mathrm{CP}$ asymmetry due to the $\delta_{C P}$ complex phase, and thus to break the parameter degeneracies. The existence of CP-violation will be tested explicitly, which is different from simply extracting the $\delta_{C P}$ value from global fits of all available data.

The probabilities of $v_{\mu} \rightarrow v_{e}$ and $\bar{v}_{\mu} \rightarrow \bar{v}_{e}$ and oscillations for $\sin ^{2} 2 \theta_{13}=0.09$ and different values of $\delta_{C P}$ and normal hierarchy $(\mathrm{NH})$ are shown in Fig. 2, as they are expected in LBNO at the $2300 \mathrm{~km}$ baseline. The plots illustrate qualitatively that the spectral information provides an unambiguous determination of the oscillations parameters and allows discriminating between the two CP-conserving scenarios. The $\delta_{C P}$ phase and matter effects introduce a well-defined energy dependence of the oscillation probability. As a consequence, the neutrino energy spectrum of the oscillated events need to be experimentally reconstructed with sufficiently good resolution in order 

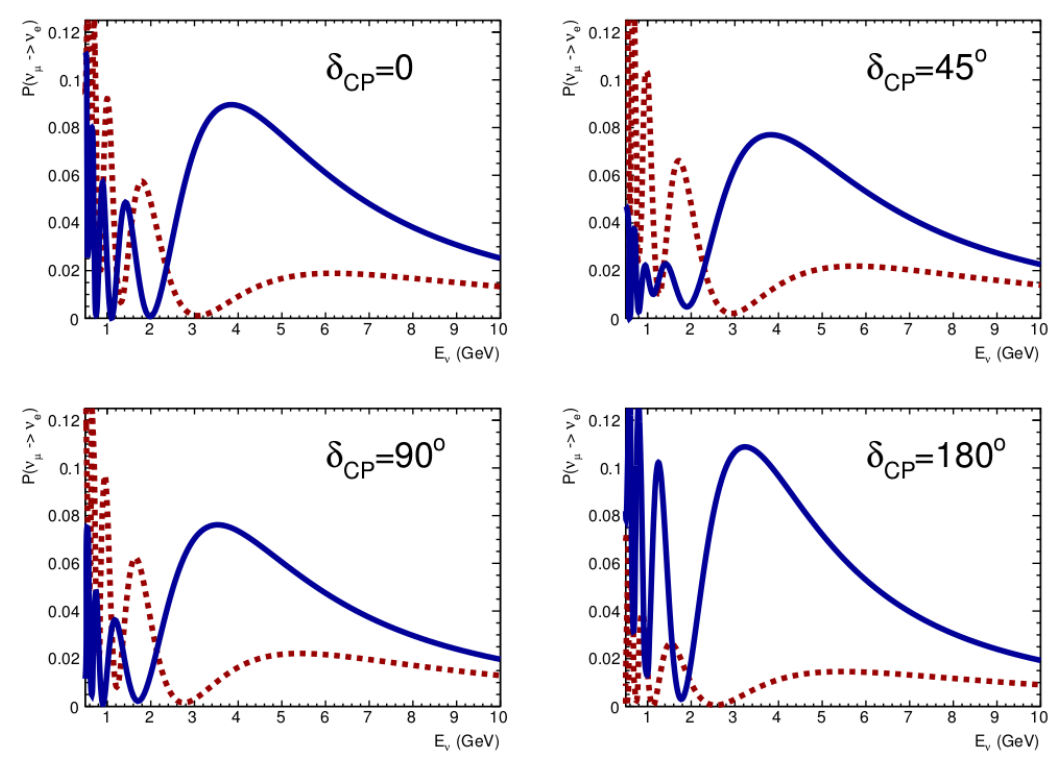

Figure 2: Oscillation probability of $v_{\mu} \rightarrow v_{e}$ and $\bar{v}_{\mu} \rightarrow \bar{v}_{e}$ for different values of $\delta \mathrm{CP}$ for normal hierarchy (NH) $\Delta m_{31}^{2}>0$ and $\sin ^{2} 2 \theta_{13}=0.09$. The spectral information provides an unambiguous determination of the oscillations parameters and allows in principle to distinguish the two CP-conserving scenarios.

to distinguish first and second maximum, and extract unambiguous information on the oscillation parameters. The spectral measurement will in addition verify the PMNS model, with possible unexpected difference between neutrinos and antineutrinos than those predicted by $\delta_{C P}$, or other non-standard deviations from the predicted $L / E$ dependence. At the same time, the matter effects at $2300 \mathrm{~km}$ are sufficiently large, that the $\mathrm{NH}$ and $\mathrm{IH}$ scenarios induce to an almost complete swap of behaviors between neutrinos. Hence, CP- and matter-induced asymmetries are totally different and distinguishable.

Preliminary sensitivity estimates to long baseline neutrino oscillations have been performed. The focus is on the sensitivity to the MH determination and the CPV discovery. The cosnidered far detector is a liquid Argon TPC with a fiducial mass of $20 \mathrm{kton}$. Two milestones for the protons-ontarget are assumed for the present studies: in the first few years of running, an integrated protonon-target intensity corresponding to $2.25 \times 10^{20}$ p.o.t. will be accumulated. This initial phase will be focused on the mass hierarchy determination and CP phase determination. In total absence of knowledge of the $\mathrm{MH}$, we take the conservative approach of 50\%-50\% sharing of the running time between neutrino and antineutrino horn focusing. Hence, we assume $1.125 \times 10^{20}$ p.o.t. in both neutrino and antineutrino mode, which should be accumulated in a few years of SPS operation (400 $\mathrm{GeV}$ proton beam energy). For the CPV search we assume an integrated pot of $1.5 \times 10^{21}$ p.o.t. and at present a $25 \%-75 \%$ sharing between neutrino and antineutrino running mode $\left(3.75 \times 10^{20}\right.$ p.o.t. and $1.125 \times 10^{21}$ p.o.t. respectively).

The goal and experimental challenge is to detect and identify charged current $v_{e} \mathrm{CC}$ (resp. $\bar{v}_{e} \mathrm{CC}$ ) interactions (Fig. 3) resulting from the subleading $v_{\mu} \rightarrow v_{e}\left(\right.$ resp. $\bar{v}_{\mu} \rightarrow \bar{v}_{e}$ ) sufficient statistics and excellent neutrino energy reconstruction resolution in order to study the $L / E$ features of the oscillation probability. In Fig. 3 is shown the expected reconstructed neutrino energy. The 
irreducible background is from the $\approx 1 \%$ intrinsic $v_{e}\left(\bar{v}_{e}\right)$ contamination of the almost pure $v_{\mu}$ $\left(\bar{v}_{\mu}\right)$ beam. This background has same final state characteristics as the signal and is therefore on an event-by-event basis indistinguishable from it. The only factor allowing to minimize this component of the background is the total neutrino energy resolution. Since this background have a much broader and rather flat energy distribution with respect to the signal, since they are dominantly produced in three-body decay processes from kaons (at high energy) and muons (at lower energies). This irreducible background will be measured in and constrained by the near detector. Another source of background is from leading $v_{\mu} \rightarrow v_{\tau}\left(\bar{v}_{\mu} \rightarrow \bar{v}_{\tau}\right)$ oscillations. Altough for the chosen baseline the oscillation probability for this transition is $O(1)$ the $v_{\tau} \mathrm{CC}$ interaction is kinematically suppressed by the required $\tau$ production thershold of about $3.5 \mathrm{GeV}$. This tranlates into a background from the $v_{\mu} \rightarrow v_{\tau} \rightarrow \tau v_{e} v_{\tau}$ process at the level of $1 \%$. Such background can be reduced by the means of a kinematical analysis based on the transverse momentum balance. The third source of contribution to the background is caused by events with no primary electrons, but where produced $\pi^{0}$ or photon conversions are mistakenly identified as electrons in neutral or muon neutrino charged current interactions. A powerful "fake" electrons rejection is an important experimental feature of the fine grain imaging provided by the LAr detector: a rejection factor of 50 on individual $\pi^{0}$ for an electron efficiency of $90 \%$ can be achieved using $d E / d x$ information and imaging properties. The $\Delta \chi^{2}$ of the mass hierarchy discriminant is presented as a function of true $\delta_{C P}$ in Fig. 4 . Both the reconstructed energy $E_{v}$ and the transverse missing momentum $p_{T}^{\text {miss }}$ distributions, in order to separate the $\tau$ interactions backgorund, are used. The signal normalization systematic error is $\pm 5 \%$, the beam electron contamination normalization is $\pm 5 \%$, the $\tau$ normalization is assumed to be poorly known due to crosssection uncertainties and are set to $\pm 50 \%$, and the $v \mathrm{NC}$ and $v_{\mu} \mathrm{CC}$ backgrounds have a systematic error of $\pm 10 \%$, and the relative norm. of positive and negative horn polarity is set to $\pm 5 \%$. In addition, a $5 \%$ bin-to-bin uncorrelated error is added. The error on the Earth density is set to $5 \%$. The obtained significance is above $5 \sigma$ over the entire range of $\delta_{C P}$ values. The CPV discovery potential is presented in Fig. 4 as a function of the $\delta_{C P}$. It exhibits the expected double peak structure with two zero values at $\delta_{C P}=0$ and $\pi$, stemming from the definition of the $\chi^{2}$, which defines the CPV discovery as the exclusion of 0 and $\pi$. With $1.5 \times 10^{21}$ p.o.t., the existence of CP-violation (CPV) can be demonstrated at the $90 \%$ C.L. for $\approx$ $70 \%$ of the $\delta_{C P}$ parameter space.

\section{References}

[1] A. Stahl et al., CERN-SPSC-2012-021 (SPSC-EOI-007).

[2] http://www.laguna-science.eu.

[3] K. Abe et al. [T2K Coll.], Phys.Rev.Lett. 107, 041801 (2011), [hep-ex/1106.2822]. P. Adamson et al. [MINOS Coll.], Phys.Rev.Lett. 107, 181802 (2011), [hep-ex/1108.0015]. Y. Abe et al. [DOUBLE-CHOOZ Coll.], Phys.Rev.Lett. 108, 131801 (2012), [hep-ex/1112.6353]. F. An et al. [DAYA-BAY Coll.], Phys.Rev.Lett. 108, 171803 (2012), [hep-ex/1203.1669]. J. Ahn et al. [RENO Coll.], Phys.Rev.Lett. 108, 191802 (2012), [hep-ex/1204.0626].

[4] V. Barger, D. Marfatia, and K. Whisnant, Phys.Rev. D 65, 073023 (2002), [hep-ph/0112119]. 

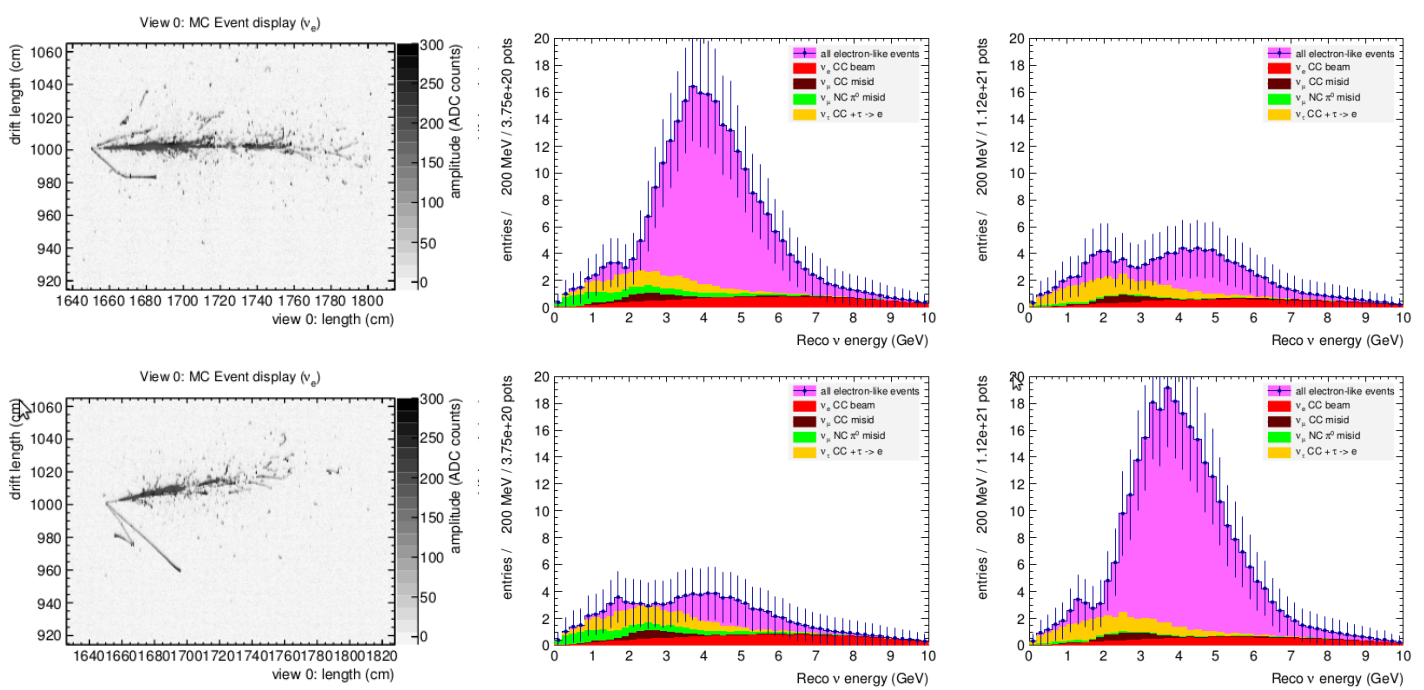

Figure 3: Left: typical $v_{e} \mathrm{CC}$ event reconstructed in the LAr far detector. Right:Reconstructed neutrino energy for electron-like final state events, showing the various components contributing to the spectrum, assuming $\delta_{C P}=0$ and (top plots) normal mass hierarchy $\mathrm{NH}$, and (bottom plots) inverted mass hierarchy IH. Neutrino-antineutrino sharing is $25 \%-75 \%$ with total p.o.t. of $1.5 \times 10^{21}$.
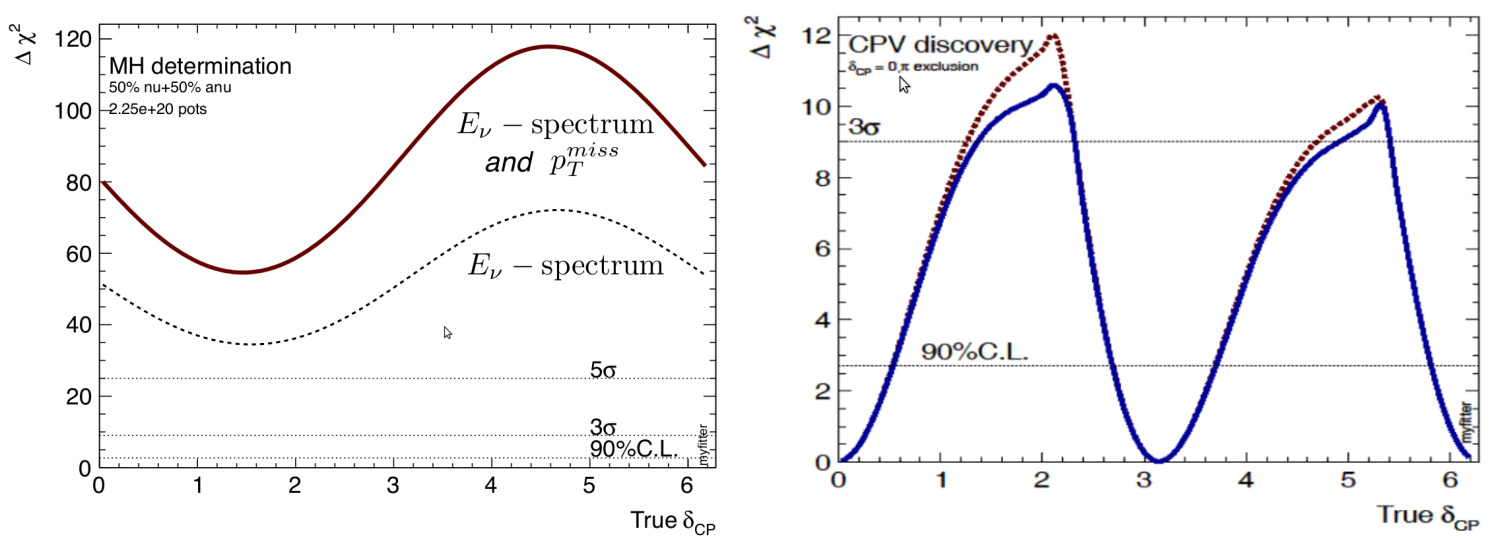

Figure 4: Left: $\Delta \chi^{2}$ of the mass hierarchy discriminant as a function of true $\delta_{C P}\left(2.25 \times 10^{20}\right.$ p.o.t., $50 \%: 50 \%$ neutrino:antineutrino beam sharing). Right: $\Delta \chi^{2}$ of the CPV discriminant as a function of true $\delta_{C P}\left(1.5 \times 10^{21}\right.$ p.o.t., $25 \%: 75 \%$ neutrino:antineutrino beam sharing). The blue curve corresponds to the case with all systematic errors included. The dashed brown curve is the case where all energy correlated errors are set to zero and the average Earth density error is reduced for $5 \%$ to $1 \%$. 